\title{
Utilização de quinonas em metodologias analíticas
}

\section{Quinones in analytic methodologies}

\author{
Isadora Souza Vieira ${ }^{1}$; Fernando César de Macedo Junior ${ }^{2}$; \\ Dimas Augusto Morozin Zaia ${ }^{3}$; Henrique de Santana ${ }^{4}$
}

\section{Resumo}

Neste artigo foi apresentado o uso de compostos quinônicos como mediadores em metodologias analíticas, baseado no monitoramento das suas reações com os analitos e a determinação de seus produtos. As potencialidades das técnicas utilizadas, bem como os aspectos mecanísticos foram discutidos. Palavras-chave: Quinonas. Análises. Caracterização.

\begin{abstract}
In this paper is showed several uses of quinonic substances presented as mediators in analytic methodologies, based on the monitoring their reactions and the detection of their products. The potentialities of different techniques as well as mechanistic features were depicted.

Key words: Quinones. Analyses. Characterization.
\end{abstract}

1 Graduando em Química, Universidade Estadual de Londrina;

Docente do Departamento de Química da Universidade Estadual de Londrina;

3 Docente do Departamento de Química da Universidade Estadual de Londrina; damzaia@uel.br

4 Docente do Departamento de Química da Universidade Estadual de Londrina; hensan@uel.br 


\section{Considerações gerais}

O desenvolvimento de metodologias analíticas sempre teve um papel de destaque nas diferentes áreas do conhecimento, sempre com ênfase nas determinações de espécies químicas em meios "reais". Pode ser destacada a análise clínica, para diagnósticos mais preciso de doenças (GANONG, 1995); na nutrição animal, visando racionalizar o uso de nutrientes (MCDONALD; EDWARDS; GREENHALGH, 1987); na tecnologia de alimentos, propondo novos produtos ou aperfeiçoamento dos existentes (FENNEMA, 1976) e na ecologia (ROBBINS, 1983), buscando a sustentabilidade na interação homem-natureza.

$\mathrm{Na}$ tentativa de resolver os problemas existentes diversas metodologias foram adaptadas visando diferentes aplicações analíticas (MURTHY; SHARMA, 1998; SURYA; MURTHY; SHARMA, 1997; HASEBE; TANAKA; UCHIYAMA, 1994; ISKANDER; MEDIEN, 1990; RZPECKI; WAITE, 1989). Dentre alguns exemplos, incluem-se a utilização de quinonas para a determinação de proteínas totais em plasma sanguíneo (ZAIA et al., 1992a; ZAIA; VERRI JUNIOR; ZAIA, 1999) ou tecidos animais (ZAIA et al., 1992b; ZAIA; VERRI JUNIOR.; ZAIA, 2000) e a determinação simultânea de aminoácidos e proteínas totais (ZAIA, et al., 1993; BARRETO; AQUINO; ZAIA, 1990); a determinação de cisteína em xampus, pílulas de alimentos, aminoácidos e a misturas de carbocisteínas em produtos farmacêuticos (ZAIA; RIBAS; ZAIA, 1999).

As metodologias analíticas têm buscado como mediadores as moléculas de quinonas, visando transformar o ambiente químico, de um contexto inacessível para os estudos científicos em colaborativos. $\mathrm{Na}$ maioria dos métodos são analisados os produtos da reação entre quinonas e a espécie a ser estudada. Os dados da literatura também têm demonstrado uma série de estudos comparativos com outros métodos utilizados correntemente, demonstrando a melhor técnica em cada situação. Como exemplo, no trabalho de Weinert, Pezza e Pezza (2008) foi determinado espectrofotometricamente o citrato de sildenafil (Viagra ${ }^{\circledR}$ ) através do p-cloranil e com a adição de peróxido de hidrogênio. Considerando a rapidez, simplicidade e baixo custo, o método aplicado na análise de formulações farmacêuticas contrapõemse às técnicas convencionais como cromatografia líquida de alta eficiência com detecção UV ou acoplada à espectrometria de massa, cromatografia eletrocinética micelar, cromatografia gasosa e eletroforese capilar, que requerem um longo tempo no preparo de amostras, maiores quantidades de solventes e a manutenção de equipamentos dispendiosos. Neste contexto, esta revisão tem como objetivo avaliar a potencialidade de alguns trabalhos envolvendo quinonas e demonstrar as diferentes ferramentas utilizadas na caracterização dos produtos de reação para a análise indireta da espécie de interesse.

A maioria dos métodos de caracterização de diferentes tipos de compostos (fármacos, aminoácidos, proteínas, entre outros) mediados por compostos quinônicos são baseados em estudos com as técnicas espectroscópicas, principalmente a absorção UV-Vis. Porém são também encontrados alguns trabalhos utilizando, com sucesso, a cromatografia líquida, fluorescência e voltametria cíclica. O uso das espectroscopias no infravermelho e Raman também têm sido empregados para a caracterização dos produtos formados.

\section{Aplicações dos compostos quinônicos em métodos analíticos quantitativos}

Na Tabela 1 são descritas algumas determinações espectrofotométricas UV-Vis de compostos de interesse e indicados os compostos quinônicos utilizados como mediadores. Dentre estes, destacamse os trabalhos discutidos a seguir.

O trabalho de Zaia, Ribas e Zaia (1999) teve como objetivo desenvolver um método sensível 
e seletivo para a determinação de cisteína ou carbocisteína em diferentes produtos comerciais. Um recobrimento de $100 \%$ foi observado igualmente para cisteína ou carbocisteína, quando o método foi aplicado para a determinação de cisteína em uma mistura de aminoácidos semelhantes ao plasma sanguíneo, xampu, pílulas alimentares, como também para a carbocisteína em produtos farmacêuticos (Mucoflux e Mucolitic). Zaia, Verri Junior e Zaia. (1999) verificaram que o método com $p$-cloranil apresentou um limite de detecção menor do que o método de Biureto $\left(52,0 \mu \mathrm{g} \mathrm{ml} \mathrm{l}^{-1}\right)$ ou com o método com a $p$-benzoquinona $(2,6-4,0 \mu \mathrm{g}$ $\mathrm{ml}^{-1}$ ). O método foi aplicado para a determinação de proteínas totais em plasma sanguíneo. Os resultados foram comparados com o método de Biureto, que é correntemente utilizado em análises clínicas. Um recobrimento de $100 \%$ tanto para soro de albumina bovina como para aminoácidos totais foi obtido quando foi utilizado $p$-benzoquinona para amostras de misturas de aminoácidos e proteínas semelhantes ao fluído cérebro espinhal (ZAIA et al., 1993).

Lorentz e Flatter (1974) descreveram um método adequado para a determinação de aminoácidos em plasma, soro e urina. Na ausência de aminas primárias, a reação foi insensível a amônia, uréia e altas concentrações de sais inorgânicos. Os dados obtidos foram comparados com os obtidos pelo método da ninidrina.
Rizk e Younis (1984) utilizaram este método para a determinação dos medicamentos em sua forma de dosagem, com boa precisão e apreciável sensibilidade. Muralikrishna, Krishnamurthy e Rao (1984) comparou os resultados obtidos para 1,4-benzoquinona na análise da piperazina com o método oficial da Farmacopéia Britânica (BP), indicando uma boa precisão do método. Medien (1996) utilizou o método para a determinação de ácido barbitúrico com três tipos de benzoquinonas e observou que os outros barbituratos não foram interferentes na reação.

Hassan, Iskander e Nashed (1985) investigaram por método cinético os efeitos sob a reação das seguintes variáveis: solvente, temperatura, concentração de quinona e presença de água. Foram obtidos resultados com recobrimento médio de $98,5 \%$ e um desvio padrão médio de 1,9\%, com nove diferentes aminas. Hasebe, Tanaka e Uchiyama (1994) monitoraram polifenóis baseandose na reação cíclica entre polifenóis e o-quinonas catalisada por Tirosinase e pelo ácido L-ascórbico.

$\mathrm{Na}$ reação redox, ocorre um aumento no $\mathrm{pH}$ devido ao consumo do próton em solução quando as o-quinonas reagem com o ácido L-ascórbico, sendo as mudanças espectrais do azul de bromotimol sensíveis a esta alteração em solução. A magnitude desta mudança no $\mathrm{pH}$ foi significantemente dependente da concentração dos polifenóis. 
Tabela 1. Algumas substâncias analisadas pelo método espectrofotométrico UV-Vis utilizando os compostos quinônicos.

\begin{tabular}{|c|c|c|c|}
\hline $\begin{array}{l}\text { Compostos } \\
\text { Quinônicos }\end{array}$ & Determinação analítica & Comentários & Referências \\
\hline p-benzoquinona & tiouréia & $\begin{array}{l}\text { Limite de detecção de } \\
\qquad 0,90 \mu \mathrm{g} / \mathrm{ml}\end{array}$ & Oliveira et al.(2004) \\
\hline $\begin{array}{l}\text { 1,2-naftoquinona-4- } \\
\text { sulfonato }\end{array}$ & $\begin{array}{l}\text { etilenoimina e } \\
\text { n-butilamina }\end{array}$ & $\begin{array}{l}\text { sensibilidade a nível de } \\
\text { ppm }\end{array}$ & $\begin{array}{l}\text { Rosenblatt, Hlinka e Epstein } \\
\text { (1995) }\end{array}$ \\
\hline$p$-benzoquinona & $\begin{array}{l}\text { drogas de sulfa } \\
\text { aminoácidos }\end{array}$ & $\begin{array}{l}* 50-124 \mu \mathrm{g} / \mathrm{ml} \\
* 1,5-34,0 \mu \mathrm{g} / \mathrm{ml}\end{array}$ & $\begin{array}{l}\text { Iskander, Medien e Nashed (1989) } \\
\text { Iskander e Medien (1990) }\end{array}$ \\
\hline$p$-benzoquinona & aminoácidos & $* \cong 2,5-4,0 \mathrm{mmol} / 1$ & Lorentz e Flatter (1974) \\
\hline$p$-benzoquinona & $\begin{array}{c}\text { cisteina } \\
\text { carbocisteina }\end{array}$ & $\begin{array}{l}* 0-40 \mu \mathrm{g} / \mathrm{ml} \\
* 0-150 \mu \mathrm{g} / \mathrm{ml}\end{array}$ & Zaia, Ribas e Zaia (1999) \\
\hline p-benzoquinona & proteínas totais & $* 4-240 \mu \mathrm{g} / \mathrm{ml}$ & Zaia et al. (1992b) \\
\hline $\begin{array}{l}\text { tetracloro- } p \text { - } \\
\text { benzoquinona }\end{array}$ & proteínas totais & $\begin{array}{l}\text { Limite de detecção de } \\
\qquad 1,25 \mu \mathrm{g} / \mathrm{ml}\end{array}$ & $\begin{array}{c}\text { Zaia, Verri Junior e Zaia, } \\
\text { (1999) }\end{array}$ \\
\hline $\begin{array}{l}\text { 2,3,5,6-tetracloro- } \\
\text { p-benzoquinona } \\
\quad \text { (cloranil) }\end{array}$ & $\begin{array}{l}\text { antibióticos } \\
\text { aminoglicosídeos }\end{array}$ & $* 1-10 \mu \mathrm{g} / \mathrm{ml}$ & Rizk e Younis (1984) \\
\hline 1,4-benzoquinona & piperazina & $* 13-120 \mu \mathrm{g} / \mathrm{ml}$ & $\begin{array}{l}\text { Muralikrishna, Krishnamurthy } \\
\text { e Rao (1984) }\end{array}$ \\
\hline $\begin{array}{l}\text { p-benzoquinona; } \\
\text { p-cloranil; 1,4- } \\
\text { naftoquinona }\end{array}$ & ácido barbitúrico & $* 0,025$ a $0,345 \mathrm{mM}$ & Medien (1996) \\
\hline p-benzoquinona & $\begin{array}{l}\text { aminas alifáticas } \\
\text { primárias e secundárias }\end{array}$ & $\begin{array}{l}\text { sem interferência de } \\
\text { aminas terciárias, } \\
\text { amônia, amidas, imidas, } \\
\text { anilidas, hidrazinas e } \\
\text { ácidos } \alpha \text {-amino. }\end{array}$ & $\begin{array}{l}\text { Hassan, Iskander e Nashed } \\
\text { (1985) }\end{array}$ \\
\hline o-quinonas & polifenóis & $\begin{array}{l}\text { Limites de detecção do } \\
\text { catecol e catecolaminas } \\
\text { (L-dopa, dopamina, } \\
\text { noradrenalina e } \\
\text { adrenalina) foram } \\
\text { da ordem de } 1 \mathrm{x} \\
10^{-7} \mathrm{M} \text { e } 1 \times 10^{-9} \mathrm{M} \\
\text { respectivamente. }\end{array}$ & $\begin{array}{c}\text { Hasebe, Tanaka e Uchiyama } \\
\text { (1994) }\end{array}$ \\
\hline
\end{tabular}

*Intervalo que a Lei de Beer-Lambert é seguida. 
Tabela 2. Substâncias analisadas por diferentes métodos utilizando os compostos quinônicos.

\begin{tabular}{|c|c|c|c|}
\hline Compostos Quinônicos & $\begin{array}{c}\text { Compostos analisados/ } \\
\text { Técnica }\end{array}$ & Comentários & Referências \\
\hline $\begin{array}{c}\text { p-benzoquinona; } \\
\text { N-cloro-p- } \\
\text { benzoquinoneimina; } \\
\text { O-[p-nitrobenzeno- } \\
\text { sulfonil] }\end{array}$ & Cianeto/ Fluorescência & $\begin{array}{l}\text { Limite de detecção de } \\
0,2 \mu \mathrm{g} / \mathrm{ml} \\
* 0,2-50 \mu \mathrm{g} / \mathrm{ml}\end{array}$ & Guilbaul e Kramer (1965) \\
\hline p-benzoquinona & Cicloserina/ HPLC & $\begin{array}{l}\text { Limite de detecção de } 10 \\
\text { ng/mL }\end{array}$ & $\begin{array}{l}\text { David, Ionescu e Dumitrescu } \\
\text { (2001) }\end{array}$ \\
\hline $\begin{array}{l}\beta \text {-naftoquinona-4- } \\
\text { sulfonato }\end{array}$ & $\begin{array}{c}\text { anfetaminas, } \\
\text { metanfetaminas e } \\
\text { outros derivados de } \\
\beta \text {-fenetilaminas/ HPLC }\end{array}$ & $\begin{array}{l}\text { Limite de detecção de } 2 \\
\mathrm{ng} / \mathrm{mL} \\
\quad * 0,25-2 \mu \mathrm{g} / \mathrm{ml}\end{array}$ & Endo et al. (1980) \\
\hline p-benzoquinona & $\begin{array}{l}\text { NADH-ácido ascórbico/ } \\
\text { Voltametria Cíclica }\end{array}$ & $* 1-10 \mathrm{mM}$ & $\begin{array}{l}\text { Murthy e Sharma (1998); } \\
\text { Surya e Murthy (1993) }\end{array}$ \\
\hline $\begin{array}{l}\text { 2-metil-5,6-dimetoxi- } \\
\text { benzoquinona }\end{array}$ & $\begin{array}{l}\mathrm{ADH} / \text { Voltametria } \\
\text { Cíclica }\end{array}$ & $* 0-12 \mathrm{mM}$ & Ikeda et al., (1997) \\
\hline p-benzoquinona & $\begin{array}{l}\text { glicose e D-Lisina/ } \\
\text { Voltametria Cíclica }\end{array}$ & $\begin{array}{l}* 1 \text { a } 50 \mathrm{mM} \text { e } 1 \text { a } 10 \\
\mathrm{mM} \text {, respectivamente. }\end{array}$ & Murthy e Sharma $(1997,1999)$ \\
\hline
\end{tabular}

*Intervalo linear.

$\mathrm{Na}$ Tabela 2 são relatados alguns casos descritos para a determinação de compostos de interesse por diferentes métodos utilizando os compostos quinônicos como mediadores.

David, Ionescu e Dumitrescu (2001) desenvolveram um método cromatográfico para determinação de cicloserina em amostras de plasma sanguíneo. O método foi baseado na derivatização da cicloserina com p-benzoquinona e quatro derivados foram obtidos. A detecção foi por fluorescência para um volume de injeção de $5 \mu \mathrm{L}$. Este método foi validado e aplicado para a determinação de cicloserina em amostras de plasma de vários voluntários saudáveis.

Murthy e Sharma (1998) utilizaram um eletrodo de grafite pirolítico modificado com benzoquinona para o estudo da atividade eletrocatalítica de oxidação do metabólico NADH e ácido ascórbico em tampão fosfato. Surya e Murthy (1993) puderam utilizar a benzoquinona como um biosensor, uma vez que a alta reversibilidade do par p-benzoquinona/ hidroquinona em um eletrodo de grafite pirolítico favoreceu a reação glicose-glicose oxidasebenzoquinona sobre este eletrodo. Ikeda et al. (1997) utilizaram um eletrodo de trabalho com pasta de carbono imobilizado com células de Acetobacter aceti. A atividade catalítica foi devido a álcool dehidrogenase (ADH) existente nas membranas citoplasmáticas. Murthy e Sharma (1997, 1999) descreveram métodos amperométricos para a análise de glicose e D-Lisina, respectivamente. Os sensores usam benzoquinona como um mediador para a transferência de elétrons entre a glicose ou D-amino ácido oxidase imobilizadas em eletrodo de grafite pirolítico e carbono vítreo, respectivamente. Foram monitorados as influências do oxigênio, $\mathrm{pH}$ e potencial de operação. 


\section{Natureza das reações}

A partir dos orbitais moleculares para 1,4benzoquinonas calculados pelo método LCAO-MO, pode ser observado que quinonas são compostos muito heterogêneos no que diz respeito à sua distribuição de densidade de elétrons $\pi$. Uma faixa extensa de valores de densidade eletrônica reflete o amplo espectro de reatividade química desta classe de moléculas (KUTYREV, 1991).

Quinonas são em alguns casos consideradas como compostos carbonílicos $\alpha, \beta$-insaturados. Entretanto, esta abordagem não é adequada uma vez que não leva em conta o caráter específico da conjugação cruzada destas estruturas.

Para um tratamento adequado da ampla reatividade química das quinonas e seus derivados deve-se ter em mente a sua grande a tendência para formar sistemas aromáticos ou semiquinonas bem como, sua habilidade para trocar substituintes do anel sem a quebra da estrutura quinona. Além disso, é importante destacar que mecanisticamente, a maioria das suas reações se processa via processos heterolíticos resultando na formação de produtos de substituição ou adição. No entanto, as quinonas estão suscetíveis também às reações homolíticas importantes.

As reações de quinonas com eletrófilos estão restritas, como regra geral, às suas interações com próton ou com agentes redutores contendo metais que transformam quinonas em hidroquinonas ou complexos de semiquinonas (MÜLLER; JOLY, 1983; FELIX; PRABHANANDA, 1984; RIVIERE; CASTEL; SATGE, 1988; ROITMAN; WONG, 1979; KUNDU, 1979). As das quinonas com nucleófilos, por outro lado são bem mais importantes.

O esquema das reações nucleofílicas pode ser representado como um processo cuja primeira etapa consiste na adição do nucleófilo $(\mathrm{Nu})$ ao anel carbônico, com subseqüente formação da estrutura (1), como observado na Figura 1. Esta etapa é comum tanto para as reações de adição nucleofílica como de substituição nucleofílica $\left(\operatorname{Ad}_{\mathrm{N}}\right.$ ou $S_{N}$ respectivamente). A diferença entre a $S_{N}$ e $\operatorname{Ad}_{N}$ ocorre nas etapas subsequentes cuja força motriz envolve a estabilização do sistema.

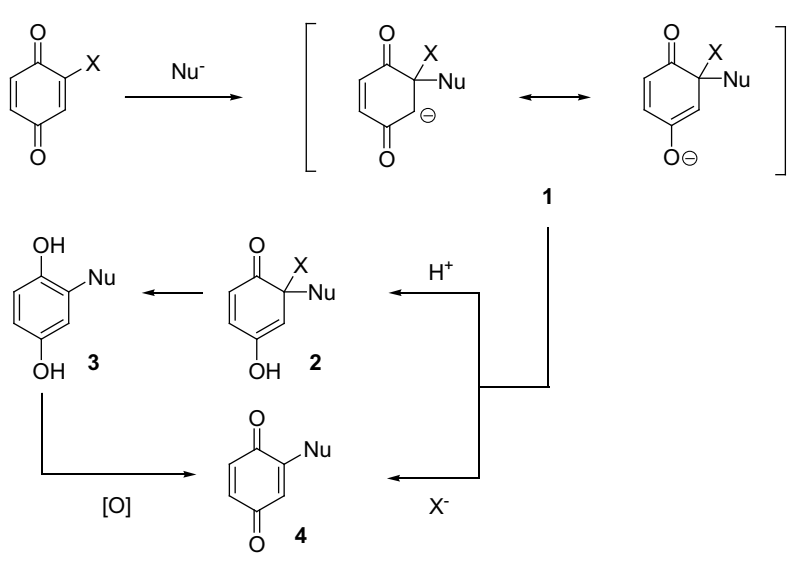

Figura 1. Esquema proposto para as reações nucleofílicas (KUTYREV, 1991).

Se um substituinte $X$ não é um bom grupo abandonador, então a adição de um contra-íon $\left(\mathrm{H}^{+}\right.$ por exemplo) ou de uma outra espécie eletrofílica é favorecida. Em seguida, o aduto (2) se isomeriza resultando em um sistema aromático como a formação da hidroquinona (3). As 1,2-quinonas reagem de maneira similar. No caso de o substituinte $\mathrm{X}$ ser um bom grupo abandonador (Hal, RO, RN) a estabilização da estrutura 1 é alcançada através da clivagem da ligação C-X com formação do ânion $\mathrm{X}$ - e da quinona (4).

Em alguns casos, um elétron pode ser transferido do reagente nucleofílico para a quinona formando um par de radicais cuja combinação resulta na estrutura intermediária (1). As hidroquinonas (3) contendo substituintes doadores de elétrons podem ainda ser facilmente oxidadas para produzir as respectivas quinonas (4), conforme ilustrado no esquema da Figura 1.

\section{Caracterização dos produtos formados}

Devido ao fato que as quinonas exibem uma variedade de reações, a caracterização formal das estruturas faz-se necessário. A molécula 
da p-benzoquinona neutra, bastante utilizada nas análises, tem sido estudadas em alguns experimentos de absorção ótica (TERHORST; KOMMANDEUR, 1979), infravermelho (BECKER; CHARNEY; ANNO, 1965; TROMMSDORFF; WIERSMA; ZELSMANN, 1985) e Raman (PALMO; PIETILA; MANNFORS, 1983). O radical cátion correspondente dap-benzoquinonatemsidoestudado por espectroscopia Raman ressonante (TRIPATHI, 1981; BECK; BRUS, 1982; SCHULER; TRIPATHI; PREBENDA, 1983) e a estrutura e as vibrações fundamentais do radical ânion foram estudados por cálculos ab Initio (CHIPMAN; PREBENDA, 1986).

Morrison, Steele e Danner (1969) mostraram que a benzoquinona forma produtos com aminas, aminoácidos, independente das cadeias laterais, e proteínas. A reação de proteínas com benzoquinona é similar à descrita para os aminoácidos. A reação do Citocromo $\mathrm{C}$ ocorre com quatro moles de benzoquinona para um mol de Citocromo C. A benzoquinona se posiciona de tal forma a não acessar os grupos heme e não causa a desnaturação ou alteração na estrutura do Citocromo C. Sastry et al. (1986) determinaram as aminoquinolinas (amodiaquina, cloroquina, primaquina e os derivados das pirimidinas, trimetoprima e pirimetamina), compostos utilizados como drogas antimalariais, baseando-se na interação destes com as quinonas (2,5-dihidroxi-3-undecil-1,4-benzoquinona; 2,5dihidroxi-1,4-benzoquinona e 2,5-dihidroxi-3,6hidroxi-1,4-benzoquinona), que promovem a formação correspondente dos complexos aceptordoador de elétrons.

Iskander, Medien e Nashed (1989) estudaram a natureza dosprodutos formados entre drogas de sulfae a p-benzoquinona. Neste trabalho a 2-sulfanilamida1,4-benzoquinona e 2,5-disulfanilamida-1,4benzoquinona foram determinadas por análise elementar e espectrofotometria no Infravermelho. Iskander e Medien (1990) propuseram que na reação entre aminoácidos e p-benzoquinona um composto monosubstituído deva ser formado, favorecido pelo rearranjo dos elétrons $\pi$ da p-benzoquinona.

El-Sayed, Mohamed e Wahbi (1986) determinaram quantitativamente a cicloserina por espectrofotometria e espectrofluorimetria, baseado na reação com p-benzoquinona. Os dados de RMN e Infravermelho do produto formado não evidenciaram a substituição no anel quinônico.

Rzepecki e Waite (1989) observaram que a reação de acoplamento entre L-prolina e os produtos de oxidação de várias catecóis servem para a identificação de catecol oxidase. O produto cromogênico, 4-N-propil-o-quinonas, foi único e estável durante a reação e determinado por método espectrofotométrico.

Segundo Bebawy et al. (1999) a interação molecular entre doador-receptor de elétrons está normalmente associada com a formação de complexos de transferência de carga, que com subseqüente dissociação do mesmo pode formar o par iônico radicalar. Barreto, Zaia e Oliveira (1994) estudou a interação entre $p$-benzoquinona e arginina em estado sólido. O produto obtido apresentou a natureza de espécie radicalar e por espectroscopia Raman ressonante foi observado que a espécie formada difere da observada quando a reação é realizada em solução. No trabalho de Oliveira et al. (2004) resultados de FT-IR do produto de reação da $p$-benzoquinona e 2,5-dicloro-p-benzoquinona com tiouréia apresentaram uma substituição no grupo sulfidrila do anel quinônico.

\section{Considerações finais}

Considerando as discussões realizadas em torno dos resultados obtidos por diferentes autores, conclui-se a viabilidade de utilização de compostos quinônicos como mediadores na determinação de compostos de interesse, visto as diferentes áreas de aplicações e as técnicas utilizadas. Verifica-se também a necessidade de experimentos envolvendo 
a caracterização destes compostos por técnicas adequadas, devidas ainda ao pouco conhecimento sobre os compostos formados.

\section{Agradecimentos}

Os autores agradecem à PROPPG/UEL pelo auxílio ao projeto no. 04853 .

\section{Referências}

BARRETO, W. J.; AQUINO, M.; ZAIA, D. A. M. A new method for total protein determination. Analytical Letters, New York, v. 23, n. 7, p. 1279-1290, 1990.

BARRETO, W. J.; ZAIA, D. A. M.; OLIVEIRA, L. F. C. The interaction of $p$-benzoquinone with amino acids in the solid state: A spectroscopic investigation. Spectrochimica Acta, Part A, v. 50, n. 66, p. 1031-1037, 1994.

BEBAWY, L. I.; EL-KOUSY, N.; SUDDIK, J. K.; SHORKY, M. Simultaneous determination of some multicomponent dosage forms by quantitative thin layer chromatography densitometric method. Journal of Pharmaceutical And Biomedical Analysis, Amsterdam, v. 21, n. 1, p. 133-142, 1999.

BECK, S. M.; BRUS, L. E. Transient raman-scattering study of the initial semi-quinone radical kinetics following photolysis of aqueous benzoquinone and hydroquinone. Journal of the American Chemical Society, Easton, v. 104, n. 18, p. 4789-4792, 1982.

BECKER, E. D.; CHARNEY, E.; ANNO, T. Molecular vibrations of quinones.6. A vibrational assignment for p-benzoquinone and 6 isotopic derivatives. Thermodynamic functions of p-benzoquinone. Journal of Chemical Physics, New York, v. 42, n. 3, p. 942-949, 1965.

CHIPMAN, D. M.; PREBENDA, M. F. Structures and fundamental vibrations of p-benzoquinone and p-benzosemiquinone radical-anion from ab Initio calculations. Journal of Physical Chemistry, Ithaca, v. 90, n. 22, p. 5557-5560, 1986.

DAVID, V.; IONESCU, M.; DUMITRESCU, V. Determination of cycloserine in human plasma by highperformance liquid chromatography with fluorescence detection, using derivatization with $p$-benzoquinone. Journal of Chromatography B., Amsterdam, v. 761, n. 1, p. 27-33, 2001.

EL-SAYED, L.; MOHAMED, Z. H.; WAHBI, A. M. Spectrophotometricand spectrofluorimetric determination of cycloserine with p-benzoquinone. Analyst, London, v. 111, n. 8, p. 915-917, 1986.

ENDO, M.; IMAMICHI, H.; MORIYASU M.; HASHIMOTO, Y.Micro-determinationofstimulant-drugs in urine by high-performance liquid-chromatography. Journal of Chromatography A, Amsterdam, v. 196, n. 2, p. 334-336, 1980.

FELIX, C. C.; PRABHANANDA, B. S. Pi-electron spin-density distributions and g-values in semiquinone cations. Journal of Chemical Physics, New York, v. 80, n. 7, p. 3078, 1984.

FENNEMA, O. R. Principles of Food Science. New York: Marcel Dekker, 1976.

GANONG, W. F. Review of Medical Physiology. San Francisco: Prentice-Hall, 1995.

GUILBAUL, G. G.; KRAMER, D. N. Specific detection and determination of cyanide using various quinone derivatives. Analytical chemistry, Washington, v. 37, n. 11, p. 1395-1399, 1965.

HASEBE, Y.; TANAKA, Y.; UCHIYAMA, S. Highly amplified spectrophotometry of polyphenols based on a cyclic reaction between polyphenols and o-quinone compounds using tyrosinase and L-ascorbic-acid. Analytical Letters, New York, v. 27, n. 1, p. 41-53, 1994.

HASSAN, S. S. M.; ISKANDER, M. L.; NASHED, N. E. Spectrophotometric determination of aliphatic primary and secondary-amines by reaction with p-benzoquinone. Talanta, London, v. 32, n. 4, p. 301-305, 1985.

IKEDA, T.; KATO, K.; MAEDA, M.; TATSUMI, H.; KANO, K.; MATSUSHITA, K. Electrocatalytic properties of Acetobacter aceti cells immobilized on electrodes for the quinone-mediated oxidation of ethanol. Journal of Electroanalytical Chemistry, Amsterdam, v. 430, n. 1/2, p. 197-204, 1997.

ISKANDER, M. L.; MEDIEN, H. A. A. Some observations on the spectrophotometric determination of amino-acids via interaction with p-benzoquinone. Microchemical Journal, New York, v. 41, n. 2, p. 172182, 1990.

ISKANDER, M. L.; MEDIEN, H. A. A.; NASHED, S. Kinetics and determination of sulfa drugs via interaction with para-benzoquinone. Microchemical Journal, New York, v. 39, n. 1, p. 43-49, 1989.

KUNDU, N. G. Convenient method for the reduction of ortho-quinones to dihydrodiols. Journal of Chemical Society, London, n. 13, p. 564, 1979.

KUTYREV, A. A. Nucleophilic reactions of quinines. Tetrahedron, v. 47, p. 8043, 1991. 
LORENTZ, K.; FLATTER, B. Simplified colorimetry of alpha-amino nitrogen in plasma, serum, or urine. Clinical Chemistry, Baltimore, v. 20, n. 12, p. 1553-1555, 1974.

MCDONALD, P.; EDWARDS, R. A.; GREENHALGH, J. F. D. Animal Nutrition. Hong Kong: Longman Scientific \& Technical, 1987.

MEDIEN, H. A. A. New method for spectrophotometric determination of quinones and barbituric acid through their reaction. A kinetic study. Spectrochimica Acta part A-Molecular and Biomolecular Spectroscopy, Amsterdam, v. 52, n. 12, p. 1679-1684, 1996.

MORRISON, M.; STEELE, W.; DANNER, D. J. Reaction of benzoquinone with amines and proteins. Archives of Biochemistry and Biophysics, New York, v. 134, n. 2, p. 515-523, 1969.

MÜLLER, P.; JOLY, D. Intermediates in the dehydrogenation of hydroaromatic compounds with quinines. Helvetica Chimica Acta, Basel, v. 66, n. 4, p. 1110-1118, 1983.

MURALIKRISHNA, U.; KRISHNAMURTHY, M.; RAO, N. S. Analytical uses of charge-transfer complexes - determination of pure and dosage forms of piperazine. Analyst, London, v. 109, n. 10, p. 1277-1364, 1984.

MURTHY, A. S. N.; SHARMA, J. Benzoquinone modified electrode for sensing NADH and ascorbic acid. Talanta, London, v. 45, n. 5, p. 951-956, 1998.

MURTHY, A. S. N.; SHARMA, J. Benzoquinonemediated biosensor for amperometric determination of D-lysine. Electroanalysis, New York, v. 11, n. 3, p. 188191, 1999.

MURTHY, A. S. N.; SHARMA, J. Benzoquinonemediated enzyme biosensor for amperometric determination of glucose. Proceedings of the Indian Academy of Sciences-Chemical Sciences, Bangalore, v. 109, n. 5, p. 295-301, 1997.

OLIVEIRA, A. N.; SANTANA, H.; ZAIA, C. T. B. V.; ZAIA, D. A. M. A study of reaction between quinones and thiourea: determination of thiourea in orange juice. Journal of Food Composition and Analysis, San Diego, v. 17, n. 2, p. 165-117, 2004.

PALMO, K.; PIETILA, L. O.; MANNFORS, B. Ramanscattering from para-benzoquinone. Journal of Molecular Spectroscopy, New York, v. 100, n. 2, p. 368-376, 1983.

RIVIERE, P.; CASTEL, A.; SATGE, J. 1,4 addition of various organohydrogermanes and organohalohydrogermanes to 3,5-di-tertbutylorthoquinone. Journal of Organometallic Chemistry, Lausanne, v. 339, n. 1/2, p. 51-60, 1988.
RIZK, M.; YOUNIS, F. Spectrophotometric assay of certain aminoglycosoide antibiotic using chloranil. Analytical letters part b-clinical and biochemical analysis, New York, v. 17, n. 6, p. 1803-1809, 1984.

ROBBINS, C. Wildlife Feeding and Nutrition. New York: Academic Press, 1983.

ROITMAN, J. N.; WONG, R. Y. Quinones and quinone methides - IV Dimerization reactions of 2-phenylmethyl5-methoxy-1,4-benzoquinones. Tetrahedron, London, v. 35, n. 9, p. 1041-1054, 1979.

ROSENBLATT, D. H.; HLINKA, P.; EPSTEIN, J. Use of 1,2-naphthoquinone-4-sulfonate for the estimation of ethylenimine and primary amines. Analytical Chemistry, Washington, v. 27, n. 8, p. 1290-1293, 1955.

RZEPECKI, L. M.; WAITE, J. H. A chromogenic assay for catecholoxidases based on the addition of L-proline to quinones. Analytical Biochemistry, New York, v. 179, n. 2, p. 375-381, 1989.

SASTRY, B. S.; RAO, E. V.; SURYANARAYANA, M. V.; SASTRY, C. S. P. Spectrophotometric determination of antimalarials using quinones. Pharmazie, Eschborn, v. 41, n. 10 , p. 739, 1986.

SCHULER, R. H.; TRIPATHI, G. N. R.; PREBENDA, M. F. Resonance raman and molecular-orbital studies of the effects of deuteration on the vibrational structure of the para-benzosemiquinone radical-anion. Journal of Chemical Physics, New York, v. 87, n. 26, p. 5357-5361, 1983.

SURYA, A.; MURTHY, N. Benzoquinone-mediated glucose glucose-oxidase reaction at pyrolytic-graphite electrode. Electroanalysis, New York, v. 5, n. 3, p. 265, 1993.

SURYA, A.; MURTHY, N.; SHARMA, J. Electrocatalytic oxidation of ascorbic acid using benzoquinone as a mediator. Electroanalysis, New York, v. 9, n. 9, p. 726, 1997.

TERHORST, G.; KOMMANDEUR, J. Singlet n-pistar states of para-benzoquinone. Chemical Physics, Amsterdam, v. 44, n. 2, p. 287-293, 1979.

TRIPATHI, G. N. R. Resonance raman-scattering of semiquinone radical-anions. Journal of Chemical Physics, New York, v. 74, n. 11, p. 6044-6049, 1981.

TROMMSDORFF, H. P., WIERSMA, D. A., ZELSMANN, H. R. Vapor solvent shift of the lowest frequency vibration of para-benzoquinone and toluquinone and the consequences for the vibrational and electronic spectral assignments. Journal of Chemical Physics, New York, v. 82, n. 1, p. 48-52, 1985. 
WEINERT, P. L.; PEZZA, L.; PEZZA, H. R. Determinação espectrofotométrica de citrato de sildenafil em formulações farmacêuticas. Química. Nova, São Paulo, v. 31, n. 1, p. 1112-1116, 2008.

ZAIA, D. A. M.; BARRETO, W. J.; SANTOS, N. J.; ENDO, A. S. Spectrophotometric method for the simultaneous determination of proteins and amino acids with $p$-benzoquinone. Analytica Chimica Acta, Amsterdam, v. 277, n. 1, p. 89-95, 1993.

ZAIA, D. A. M.; OBARA, M. M.; ROCKENBACH, S.R.; BARRETO, W. J.; GAZIRI, L. C. J.; ZAIA, C. T. B. V.; LICHTIG, J. Spectrophotometric determination of total proteins in blood-plasma with parabenzoquinone. Brazilian Journal of Medical and Biological Research, Curitiba, v. 25, n. 3, p. 549, 1992 a.

ZAIA, D. A. M.; RIBAS, K. C. L.; ZAIA, C. T. B. V. Spectrophotometric determination of cysteine and/or carbocysteine in a mixture of amino acids, shampoo, and pharmaceutical products using $p$-benzoquinone. Talanta, London, v. 50, n. 5, p. 1003-1010, 1999.
ZAIA, D. A. M.; ROCKENBACH, S. R.; OBARA, M. M.; BARRETO, W. J.; ARIZAWA, S.; CURI, R.; LICHTIG, J. Protein measurement in several rat-tissues by para-benzoquinone (pbq) method - a comparativestudy with Lowry method. Analytical Letters, New York, v. 25, n. 7, p. 1225-1234, 1992 b.

ZAIA, D. A. M.; VERRI JUNIOR., W. A.; ZAIA, C. T. B. V. Determination of total proteins: a study of reaction between quinones and proteins. Talanta, London, v. 49, n. 2 , p. 373-376, 1999.

ZAIA, D. A. M.; VERRI JUNIOR., W. A.; ZAIA, C. T. B. V. Determination of total proteins in several tissues of rat: a comparative study among spectrophotometric methods. Microchemical Journal, New York, v. 64, n. 3, p. 235-239, 2000. 